\title{
THE IMPACT OF MEMS ON THE CHEMICAL AND PHARMACEUTICAL INDUSTRIES
}

\author{
Klavs F. Jensen \\ Departments Chemical Engineering and Materials Science and Engineering \\ Massachusetts Institute of Technology \\ Cambridge, MA 02139
}

\begin{abstract}
Microfabrication techniques are increasingly used in different fields of chemistry to realize structures with capabilities exceeding those of conventional macroscopic systems. In addition to already demonstrated analysis applications ( $\mu$ TAS), MEMS microreactor systems are expected to have a number of advantages for chemical synthesis, chemical kinetics studies, and process development. Current efforts in microfabrication and application of microchemical systems are reviewed. Chemical processing advantages from increased heat and mass transfer in small dimensions are demonstrated with model gas, liquid, and multiphase reaction systems. These case studies also serve to illustrate benefits of integrating sensors for flow, temperature, and chemical composition with microfluidic reaction and control components. The role of process simulations in the development of microreaction technology is included along with approaches to scale up via replication of microchemical devices.
\end{abstract}

\section{INTRODUCTION}

Microreactors are sub-millimeter-scale, integrated chemical systems built by microfabrication methods generally used for MEMS [1], LIGA [2, 3], mechanical micromachining [2, 3], and soft lithography methods $[4,5]$. Microchemical systems have feature sizes in the micron to hundreds of micron range and may be integrated with sensors and actuators. The reduction in size and integration of multiple functions creates structures with capabilities that exceed those of conventional macroscopic systems, adding new functionality while potentially making low cost mass production possible.

Miniaturization of chemical analytic devices in "micro-totalanalysis-systems" ( $\mu \mathrm{TAS})[6]$ represents a natural extension of MEMS technology to chemistry and biology with obvious applications in combinatorial chemistry, high throughput scrcening, and portable analytical measurement devices. The merging of $\mu$ TAS techniques with microreaction technology promises to yield a wide range of novel devices for high throughput screening, as well as on-line monitoring of production systems.

Microreaction technology is also expected to have a number of advantages for chemical production $[3,7]$. The high heat and mass transfer rates possible in microfluidic systems allow reactions to be performed under more aggressive conditions with higher yields than can be achieved with conventional reactors. More importantly, new reaction pathways deemed too difficult in conventional microscopic equipment, e.g, direct fluorination of aromatic compounds [8], could be pursued. Even if a microreactor fails, the small quantity of chemicals released accidentally could be easily contained. Moreover, the presence of integrated sensor and control units could allow the failed reactor to be isolated and replaced while other parallel units continued production. These inherent safety characteristics suggest that production scale systems of multiple microreactors should enable distributed pointof-use synthesis of chemicals with storage and shipping limitations, such as highly reactive and toxic intermediates (e.g., cyanides, peroxides, azides). As a demonstration of these concepts, DuPont has synthesized a number of potentially hazardous chemicals, including isocyanates, in a microreactor formed by bonding silicon wafers patterned to form channels, preheaters, and catalytic reactor sections [7].

Scale-up to production by replication of microreactor units used in the laboratory would eliminate costly redesign and pilot plant experiments, thereby shortening the development time from laboratory to commercial production. This approach would be particularly advantageous for the fine chemical and pharmaceutical industries, where production amounts are often small-less than a few metric tons per year. The strategy would also allow for scheduled, gradual investment in new chemical production facilities without committing to a large production facility from the outset.

Microchemical systems for combinatorial synthesis and screening of small molecules and systems for nucleic acid synthesis and detection have already revolutionized drug discovery in pharmaceutical companies. Rapid screening of catalysts and product synthesis pathways could lead to analogous productivity increases in chemical industry laboratories. Experimentation at the conventional bench scale is limited by high costs of reagents and safety concerns that the small volumes and inherent safety characteristics of microreactors could effectively eliminate. The introduction of new chemicals is also limited by the risk and high capital costs of scaling from laboratory to production plant. The ability to scale-up by replication allows a gradual scale up with input from customers and no reactor redesign.

Chemical laboratories already use small reactors for testing process chemistries; for example, catalyst testing often uses small tubular reactors formed by filling powdered catalysts into glass tubes. However, the overall procedure is faced with bench top analytical equipment, large panels of complex fluid handling manifolds, and containment vessels. Chemical detection is the rate-limiting step in most methods since detailed product information must be obtained using sequential screening. With the continual advances in $\mu \mathrm{TAS}$ and microfabrication techniques, these macroscopic test systems could eventually be replaced by PC-card sized microchemical systems consisting of integrated microfluidic, sensor, control, and reaction components. Such systems would clearly require less space, utilities, and produce less waste. They would enable high-throughput screening of process chemistries under controlled conditions, which is often difficult in conventional macroscopic systems. Moreover, the small dimensions imply laminar flow, making it feasible to fully characterize heat and mass transfer and extract chemical kinetic parameters from sensor data. The high heat and mass transfer rates possible in microfluidic systems also allow reactions to be performed under more uniform temperature conditions. By using the control of fluid contacting patterns afforded by MEMS 
fabrication techniques, it should become possible to design reactors that achieve specific research and development objectives.

\section{MICROREACTOR DESIGN AND FABRICATION}

Current microreactor designs depend on the target applications, which can be broadly classified in order of increasing complexity as homogeneous gas-phase reactions, heterogeneous catalytic gas-phase reactions, liquid-liquid reactions (both miscible and immiscible systems), heterogeneous liquid phase reactions, and multiphase (gas-liquid-solid) reactions. Fluid flow combined with mass and heat transfer (transport phenomena) are easiest to handle in the case of two gases reacting without the solid catalyst, but such systems are rare. Most chemical systems of commercial interest require the presence of a catalyst-either as a thin film on a solid surface or dispersed on a high surface area porous support. Mixing is often a critical issue in liquid phase reactions and the laminar flows characteristics of microreactors imply that the turbulent mixing typically realized in macroscopic equipment has to be replaced by effective mixing by diffusion across thin liquid layers. The contact pattern for reaction components becomes especially important in the case of multiphase reactions, such as the hydrogenation of an unsaturated hydrocarbon over a solid catalyst. Efficient mixing and access to catalytic sites can lead to orders of magnitude enhancement in performance, which suggests that the precise control offered by microfabrication could have particular opportunities in multiphase reactions. Besides this application, microreaction technology is most likely to be successful for fast reactions, systems requiring precise control of reagent contacting, and for novel reaction pathways that are difficult to realize on a macroscopic scale.

Current microreactor fabrication methods fall into four main categories:

(i) MEMS based,

(ii) LIGA derived,

(iii) patterned laminated structures, and

(iv) soft lithography based polymer devices.

MEMS based devices are based on classical bulk machining and deep reactive ion etching (RIE) + bonding approaches. Recent work at MIT serves as examples in the following sections. Forschnungszentrum Karlsruhe and the Institute for Micro technology Mains (IMM) have been the primary drivers of LIGA derived microreactors, micromixers, and micro-heat-exchangers. These devices typically consist of multiple layers of multichannels fabricated in metals. Coating of the interior surface of these channels with a catalytic active material turns mixers and heat exchangers into reactors. The devices have been demonstrated for a number of model gas-surface catalytic reactions, liquid phase, and gas-liquid reactions, as well as selected commercial demonstrations. The latter includes a partial oxidation reaction of a vitamin intermcdiate, an addition reaction for fine chemicals production, and polymer synthesis. Similar devices have been fabricated using microelectro discharge machining ( $\mu$ EDM) techniques. Reactors have also been fabricated in polymers by microinjection molding, and embossing techniques.

Pacific Northwest National Laboratories (PNNL) has pioneered microfabrication by lamination of multichannel sheets. Each sheet of metal, ceramic, or plastic is pattemed by a variety of techniques including photolithography, $\square E D M$, etching (ion or chemical), and laser ablation. The sheets are then laminated to form the final microchannel reactor. Devices have been demonstrated for energy related applications, specifically fuel evaporation and reforming $[9,10]$.
Lamination of patterned ceramic tapes can also be used to create microreactor components. Laminating multiple layers machined with desired patterns again makes three-dimensional device structures. In the "green state," ceramic tapes are soft, pliable, and easily machinable. A well-developed thick film technology exists for deposition of various metals and electrical components on the tapes in the pre-fired state and the formation of three-dimensional interconnects.

"Soft lithography" refers to a collection of techniques pioneered by Whitesides and his coworkers $[4,5]$. The common feature in these methods is the use of a pattern transfer element that is a transparent elastomer, usually poly(dimethylsiloxane) PDMS, having a pattern embossed on its surface. PDMS is suitable for aqueous systems, but it swells in many organic solvents and has limited temperature stability. Therefore, PDMS based microreaction components have seen limited biological applications, including fluid mixers and electrophoresis. However, when combined with electroplating of metals and molding of ceramics, the rapid prototyping advantages of soft lithography have considerable applications in microreaction technology. Examples of this approach are included in the following discussion of MEMS based microreactors.

\section{GAS-PHASE REACTORS}

A common microreactor design used for gas-phase heterogeneous reactions is a microchannel device that can be integrated with a heat exchange layer for highly exothermic reactions [9-11]. These devices have the advantage of high productivity per unit volume, but they suffer from lack of sensing and active control within the microchannel assembly-similar to conventional ceramic monolith reactors. Thin walled microreactors offer the opportunity for the integration of heaters, as well as flow and temperature sensors on the external side of the reaction region (see Figure 1). The micron-thick wall provides good thermal contact with the catalyst in the interior and the low thermal mass of the thin wall has the further advantage of fast $(\sim 10$ ms) thermal response times.

The catalyst can be deposited on the interior wall by a variety of techniques including thin film deposition by physical or chemical vapor deposition and liquid preparation techniques. Thin film deposition techniques of the catalyst are typically limited to metals and simple oxides. Moreover, the resulting film has a low surface area and must be roughened by thermal activation. Wet preparation techniques allow the creation of catalyst systems similar to those used in conventional reactors, but surface tension effects cause the catalyst solution to collect in channel corners. Aerosol sprays or ink jet printing allows placement of the catalyst on a desired region of the membrane.

Oxidation and partial oxidation reactions of a number of compounds, including hydrogen, ammonia, and ethane, have been successfully carried out in the flammable regime [13]. The ability to control the catalyst temperature, and therefore the selectivity, of partial oxidation reactions is a critical factor determining reactor performance. During an exothermic reaction, the heat is supplied to the catalyst wall by both the heaters and the reaction. The primary heat loss mechanism is through conduction in the thin top wall to the reactor bulk silicon side walls. Therefore, by varying the thickness and thermal conductivity of the top wall (by using SOI wafers with varying silicon thickness), the ability of the reactor to dissipate the heat of the reaction can be controlled (see Figures $2 \mathrm{a}$ and $\mathrm{b}$ ). This feature provides access to a wider temperature window of operating conditions and milder oxidation conditions than can be achieved in conventional systems. 

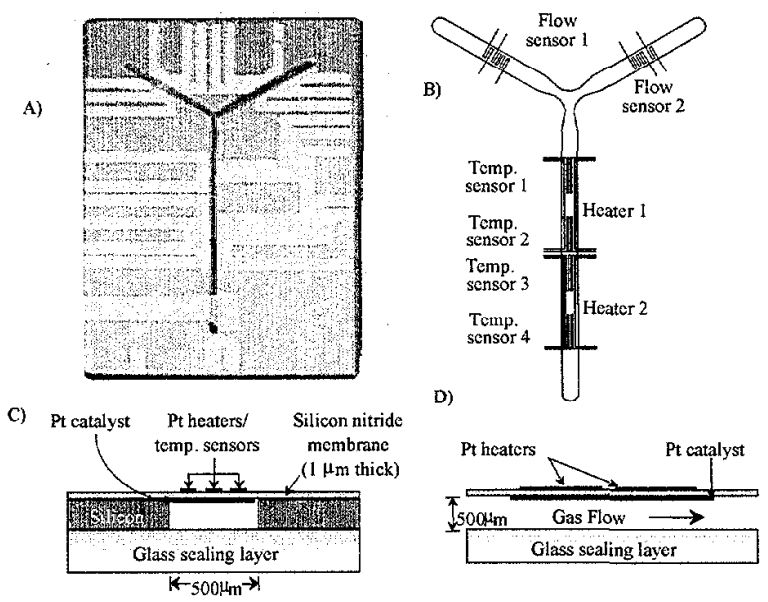

Figure 1 Gas-phase microreactor. (A) Photograph of device. (B) Top view schematic. (C) Side view across channel. (D) Side view along flow direction [12].
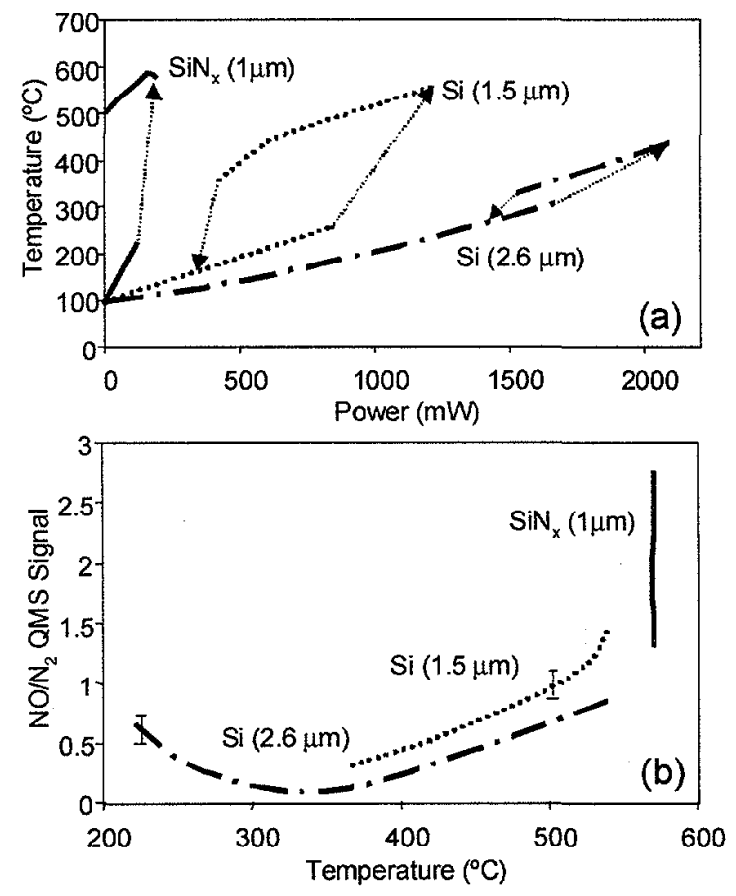

Figure 2 (a) Ignition'extinction behavior with varying input power for ammonia oxidation over platinum in microreactors with different wall materials and thickness. (b) Corresponding variation in selectivity towards nitrogen and nitrogen monoxide.

The use of a permeable membrane allows the integration of separation with chemical reaction, as in macroscopic membrane reactors [14]. For example, the integration of a submicron-thick palladium membrane makes a highly efficient hydrogen purification device and provides the potential for conducting hydrogenation and dehydrogenation reactions [15]. This approach also allows the use of other membrane materials for highly selective gas separation. Alternatively, the holes in the support structure can be used to disperse gas into a liquid stream or can act as a particle filter.

\section{LIQIUD-PHASE REACTORS}

Mixing is a critical issue in the design of liquid phase microreactors. The small dimensions in microfluidic devices imply small Reynolds numbers and laminar flow so that mixing occurs by diffusion. This characteristic becomes both a challenge and an advantage for liquid phase reaction systems. The slow mixing of co-flowing streams offers additional opportunities for phase transfer reactions and separation devices $[16,17]$, and it can be exploited in novel fabrication schemes [18].

Mixing is generally accomplished by the repeated lamination of the two streams to be mixed in order to increase the contact area and reduce diffusion lengths [19]. Ultimately, the design of a micromixing unit is a trade-off between mixing speed, pressure drop, volume flow, feasibility of microfabrication, and integration with chemical detection devices.

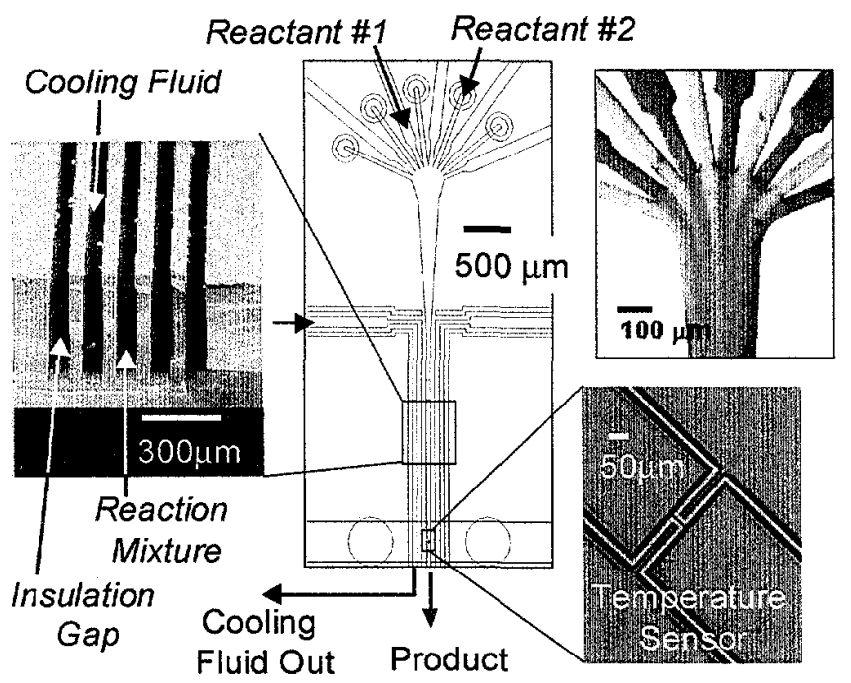

Figure 3 Liquid phase reactor with lamination of fluid streams and integrated with heat exchangers and temperature sensors in downstream reaction zone. Upper right hand insert shows mixing of acid base mixture in gray tone image of indicator color.

Figure 3 shows an example of a microfabricated liquid phase reactor that integrates laminar mixing, hydrodynamic focusing, rapid heat transfer, and temperature sensing. The integrated reactor was fabricated using SIO wafers. DRIE [20] was used to form channels $50-400 \mu \mathrm{m}$ wide and $\sim 500 \mu \mathrm{m}$ deep. An anodically bonded Pyrex wafer capped the wafer. Model studies with acidbase reactions show that the reactor achieves complete mixing in $\sim 10 \mathrm{~ms}$ depending on the fluid properties. The relatively high transfer coefficient $\left[1500 \quad \mathrm{~W} /\left(\mathrm{m}^{2} \cdot \mathrm{C}^{\circ}\right)\right]$ realized in the microfabricated heat exchanger provided excellent cooling of even highly exothermic reactions such as hydrolysis of propionyl acid chloride.

\section{MULTIPHASE REACTORS}

Gas-liquid-solid reactions are ubiquitous throughout the chemical industry and provide unique opportunities for microfabrication. The high surface-to-volume ratios attainable in microfabricated structures, leading to improved thermal management and fast mass transfer, suggest that microfabricated multiphase systems could have performance advantages relative to conventional macroscopic systems. We have demonstrated these 
characteristics for single and multichannel, multiphase packed bed reactors (Figure 4), again fabricated using deep RIE technology and bonding techniques $[21,22]$. In addition to the microfluidics, thin-film resistive heaters and temperature sensors have been integrated with the device. Each reaction chamber holds porous catalyst particles in place by a microfabricated filter as reactants are fed continuously in a co-current fashion. The gas and liquid reactant streams are brought into contact by a series of interleaved, high-aspect ratio inlet channels $(25 \mu \mathrm{m}$ wide $\times 300 \mu \mathrm{m}$ deep $)$ designed to increase gas-liquid mass transfer. The catalyst restrainer is formed within the reaction channel by etching a series of posts $40 \mu \mathrm{m}$ wide separated by $25 \mu \mathrm{m}$ gaps.

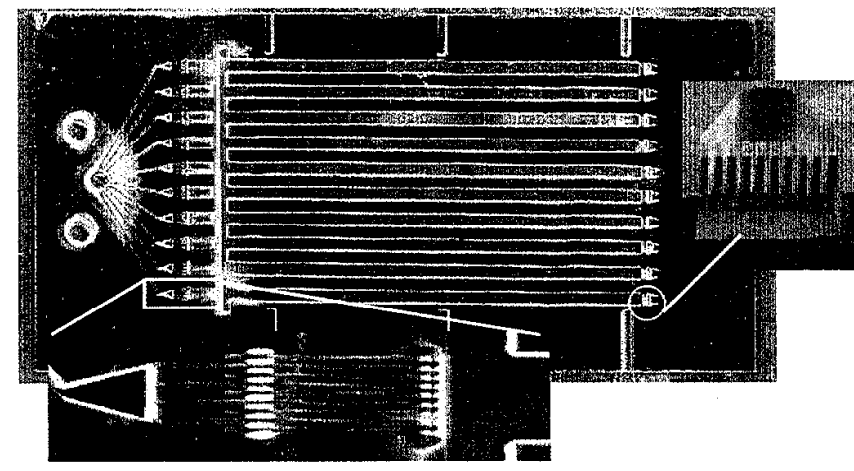

Figure 4 Multichannel multiphase packed bed reactor [22].

Model reaction studies (including hydrogenation of cyclohexene and oxidation of benzyl alcohol) over catalyst particles have been used to characterize the mass transfer and reaction behavior of the multiphase microreactor. As in the case of macroscopic reactors, a bubble flow regime is observed at low gas flow rates; pulsing flow occurs at high gas flow rates, and a steady co-current flow is found at intermediate conditions. These results demonstrate that basic multiphase flow concepts extend to microfluidic systems.

Microfabrication also provides unique opportunities for creating catalyst support systems that avoid packing variations associated with distribution of catalyst particle sizes. For example, posts can be formed by DRIE and rendered catalytic by wash coats and surface chemistry treatments. Similar structures have been developed for chromatography and concentration of DNA [23].

Selected multiphase systems have been investigated. Oxidation reactions, exemplified by the oxidation of benzaldehyde, have been demonstrated in a single-channel reactor system. In this case, the inherent safety of a small reaction volume $(4 \mu \mathrm{l})$ allows the reaction of pure oxygen and organic solvents at elevated temperatures. The hydrogenation of cyclohexene has been used to characterize the mass transfer coefficient of the multiple channel reactor using traditional reaction engineering analysis. Values of the mass transfer coefficient for the multiple channel microreactor have been determined to be two orders of magnitude larger than those reported for typical macroscopic reactors. Therefore, for reactions that operate in a mass-transfer limited regime, microreaction devices could be considerably smaller in volume and still maintain high productivity.

\section{INTEGRATION OF CHEMICAL SENSORS}

The determination of chemical products and their amounts is often a time-consuming challenge in chemical research and development. In order for microreaction technology to be successful it must include chemical sensors. Having a small reactor interfaced to large bench top analytical equipment often implies that external, fluid chamber volume is much larger than the volume of the microreactor. This discrepancy raises the potential that the observed reactor volume is the reactor-analytical system interface, instead of the microreactor. The problem is avoided only if (i) the reaction reaches full conversion in the microreactor, (ii) the reactants and products are separated in the microreactor, or (iii) the reaction is quenched in the microreactor.

In the case of liquid phase reactions that can be monitored by color changes or variations in the UV spectrum, the reactor can readily be interfaced to an optical fiber light source and detector to enable on-line monitoring. However, UV-visible spectroscopy has limited chemical resolution, i.e., features are broad and common for many compounds.

Infrared (IR) detection is a broadly applicable detection method that can be integrated with liquid phase microreactors by using the IR transparency of silicon. Thickness, doping of the wafer, as well as reflections from the substrate surface, adversely affect transmission. Although the $40 \%$ transmission obtained using uncoated samples is sufficient for most applications, one can apply an anti-reflective (AR) coating to increase silicon transmission [24].

The short path lengths and optical densities usually imply that IR absorption spectroscopy is not practicable in gas-phase microreactors. As an alternative optical method for gas-phase detection, photoacoustic spectroscopy (PAS) offers several advantages. In photoacoustic spectroscopy, incident light is modulated at an acoustic frequency. If the optical wavelength couples to an energy transition in the gas, the gas absorbs the light resulting in a periodic gas expansion. PAS applies to many chemical compounds and its sensitivity scales inversely with dimensions. The recent detection of propane in a microfabricated photoacoustic cell illustrates the potential of the spectroscopy for technique in microscale chemical analysis [25].

\section{COMBINING MEMS AND SOFT LITHOGRAPHY}

In many cases, silicon functions well as the substrate for a microchemical reactor, but different materials (e.g. polymers) that have desirable material properties may be preferable for some application [26]. The photosensitive epoxy SU-8 [27] offers opportunities for producing hybrid microchemical devices in silicon and SU-8, quartz and SU-8, and entirely from SU-8. In all cases silicon, quartz, or pyrex function as the substrate on which the device is formed. In some cases, the device is used as is, and in other cases it is released from the substrate. SU-8 can be used to create sealed microfluidic channels, to bond materials and to planarize structures. The ability to planarize allows patterned metal structures (for electrochemical detection/synthesis or for heating) to be incorporated into reaction chambers. Figure 5 shows an electrochemical reactor in which the electrodes and fluidic interconnects were formed on a silicon substrate and the fluidic channels were formed using an SU-8 bonding approach. The flexibility creates additional opportunities for liquid-phase microreactors. At low temperatures, for example, it becomes feasible to fabricate composite quartz/SU-8 devices that allow for in situ UV detection through a quartz window.

\section{SIMULATION OF MICROREACTOR SYSTEMS}

An in-depth understanding of the operating characteristics of microreactors is necessary to evaluate the benefits and disadvantages associated with new microreactor designs. Several 
commercial software tools are available to quantify different aspects of microfabricated devices, but they are not universally applicable [28, 29]. Simulations serve not only as a design tool, but also as a means to interpret experimental data [30]. Models have been used to evaluate design changes such as changing the top wall material and thickness, as described above, heater design, [31], and flow meter design [12] in the gas-phase reactor. Computational fluid dynamic software also provides insight into liquid-phase mixing. In particular, the use of simulation allows inclusion of three-dimensional wall effects often neglected in liquid layer mixers. If all physical parameters are known, simulations should accurately reflect experimental observations since the basic transport equations for laminar flow are well established. Thus, the computational approach to new designs can avoids costly, iterative experimental design process where components are fabricated, tested, and then redesigned to improve performance.

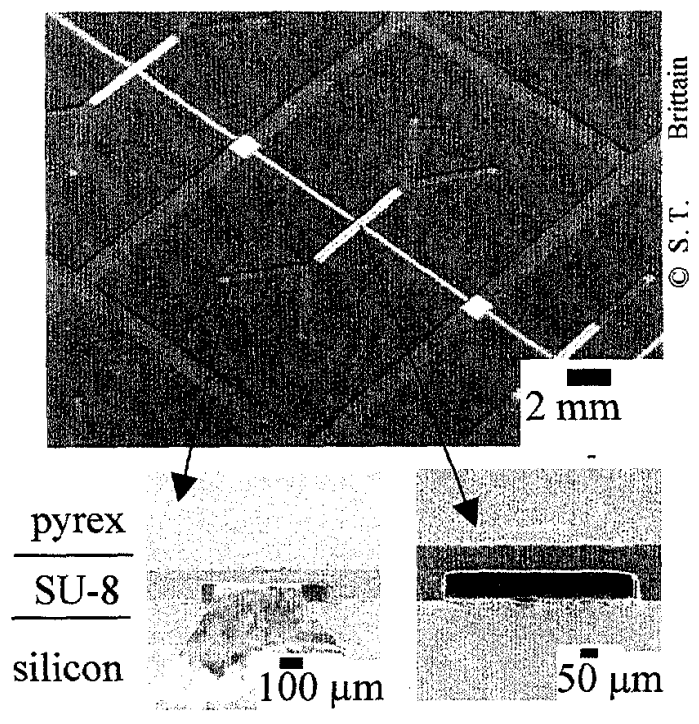

Figure 5. Photograph of electrochemical microreactor and SEM images showing cross-sections through the devices.

\section{SCALE-UP}

In the past, the microreaction community has focused on the design of individual microreactors. No significant efforts have been made to build parallel arrays of reactors as originally envisioned by early papers in the field. The concept of scale-up by replication of many units (scale-out) at first glance may appear to be simple, but the strategy presents new challenges that have not been addressed in previous chemical system scale-up. Particularly, the areas of reactor monitoring and control become increasingly complex as the parallel array size grows to a large number of reactors.

To address issues in microreactor scale-up, MIT and DuPont have begun construction of a multiple reactor test station for gasphase systems [32]. The fabrication of this system serves as a test case for the development of more complex microreactor based systems. The approach addresses the difficulties in developing electrical and fluidic interfaces to microreactors that must be interchanged frequently, have heated outlet lines, and require millisecond real-time control algorithms. The multiple microreactor test station (Figure 6) not only includes microfabricated reactors, but also integrates othor MEMS components for fluidic control. This system contains all the components of a conventional catalyst test station (feed gas manifold, reactor feed manifold, reactors, and control circuitry), but with a dramatically smaller footprint. It represents the first demonstration of a parallel microreactor array that integrates reactors with fluidic distribution and control components in a scalable fashion.

\section{CONCLUSIONS}

The examples represent shown here a small fraction of the many designs for microreactors being pursued or envisioned by different research groups. In developing microreaction technology, it will be essential to focus on systems where microfabrication can provide unique process advantages. Such advantages could be derived from increased mass and heat transfer, leading to improved yield and safety for an existing process. The real value of the miniaturization effort, however, would be in exploring new reaction pathways and finding economical and environmentally benign solutions to chemical manufacturing. It will be important to exploit characteristics resulting from the small dimensions beyond the high transport rates, specifically forces associated with high surface area-to-volume ratios. In order for microreactors to move beyond the laboratory into chemical production, they must also be integrated with sensors and actuators, either on the same chip, or through hybrid integration schemes. The integration of chemical systems with sensors in $\mu \mathrm{TAS}$ is already rapidly expanding the field, and cross-fertilization with microreactors for chemical synthesis will ultimately result in integrated chemical processors. The packaging of multiple reactors presents significant challenges in fluid handling, local reactor monitoring, and control. Thus, the realization of microreaction technology will require multidisciplinary research efforts in collaboration with users of the technology.

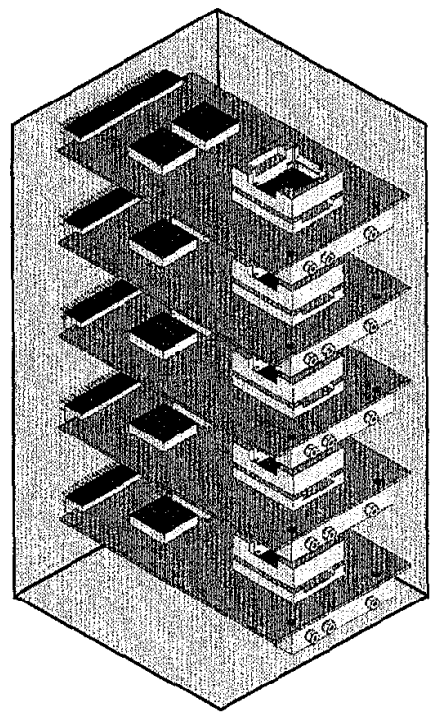

Figure 6. Conceptual drawing of packaged, parallel microreactor system [32].

\section{ACKNOWLEDGEMENTS}

The author thanks Martin A. Schmidt, the MIT microreactor group, and James F. Ryley and collaborators at DuPont for stimulating research interactions, and the DARPA MicroFlumes Program (F30602-97-2-0100) for financial support. 


\section{REFERENCES}

1. K. D. Wise, "Special issue on Integrated Sensors, Microactuators, and Microsystems (MEMS)", Proceedings of the IEEE, 86, 1531 (1998).

2. W. Ehrfeld, C. Grärtner, K. Golbig, V. Hessel, R. Konrad, H. Löwe, T. Richter, and T. Schulz, "Fabrication Of Components and Systems for Chemical and Biological Microreactors", in Microreaction Technology, W. Ehrfeld, Editor Springer, Berlin (1998), 72-90.

3. W. Ehrfeld, V. Hessel, and H. Lehr, "Microreactors for Chemical Synthesis and Biotechnology - Current Developments and Future Applications", in Microsystem Technology in Chemistry and Life Science (1998), 233-252.

4. Y. N. Xia and G. M. Whitesides, "Soft Lithography", Ann.Rev. Mat. Sci, 28, 153 (1998).

5. Y. N. Xia and G. M. Whitesides, "Soft Lithography", Ang. Chem-Int. Ed. 37, 551 (1998).

6. A. van den Berg and D. J. Harrison, "Micro Total Analysis Systems"98", Micro Total Analysis Systems $\mu 1$ 'AS '98, eds. Kluwer Academic Publishers, Dordrecht (1998).

7. J. J. Lerou, et al., "Microfabricated Minichemical Systems: Technical Feasibility", in Microsystem Technology for Chemical and Biological Microreactors: Papers of the Workshop on Microsystem Technology, Mainz, 20-21 February, 1995, DECHEMA, Frankfurt (1996), 51-69.

8. R. D. Chambers and R. C. H. Spink, "Microreactors for Elemental Fluorine", Chem. Commun., 883 (1999).

9. A. Y. Tonkovich, J. L. Zilka, M. J. LaMont, Y. Wang, and R. S. Wegeng, "Microchannel Reactors for Fuel Processing Applications. I. Water Gas Shift Reactor", Chem. Eng. Sci., 54, 2947 (1999).

10. T. A. Ameel, R. O. Warrington, R. S. Wegeng, and M. K. Drost, "Miniaturization Technologies Applied to Energy Systems", Energy Conv. Management, 38, 969 (1997).

11. G. Wießmeier and D. Hönicke, "Microfabricated Components for Heterogeneously Catalysed Reactions", J. Micromech. Microeng, 6, 285-289 (1996).

12. D. J. Quiram, K. F. Jensen, M. A. Schmidt, P. L. Mills, J. F. Ryley, and M. D. Wetzel. "Integrated Microchemical Systems: Opportunities for Process Design" in Foundations of Computer Aided Process Design. 1999. Colorado, CACHE, Colorado (1999),

13. R. Srinivasan, I.-M. Hsing, P. E. Berger, K. F. Jensen, S. L. Firebaugh, M. A. Schmidt, M. P. Harold, J. J. Lerou, and J. F. Ryley, "Micromachined Reactors for Catalytic Partial Oxidation Reactions", AIChE Journal, 43, 3059 (1997).

14. R. Govind and N. Itoh, "Membrane Reactor Technology", AIChE Symposium Series, 268, AIChE, New York, (1989).

15. A. Franz, K. F. Jensen, and M. A. Schmidt. "Palladium Based Micromembranes for Hydrogen Separation and Hydrogenation/Dehydrogenation Reactions" in 12th International Conference on MicroElectro-Mechanical Systems. Orlando, Florida, IEEE (1999), 382-385.

16. J. P. Brody, P. Yager, R. E. Goldstein, and R. H. Austin, "Biotechnology at Low Reynolds Numbers", Biophysical Journal, 7l, 3430 (1996).

17. J. R. Burns and C. Ramshaw, "Development of a Microreactor for Chemical Production", Trans IChemE, 77, 206 (1999).

18. P. J. A. Kenis, R. F. Ismagilov, and G. M. Whitesides, "Microfabrication Inside Capillaries Using Multiphase Laminar Flow Patterning", Science, 285, 83 (1999).
19. W. Ehrfeld, K. Golbig, V. Hessel, H. Lowe, and T. Richter, "Characterization Of Mixing In Micromixers By a Test Reaction: Single Mixing Units and Mixer Arrays", Ind. Eng. Chem. Research, 38, 1075 (1999).

20. A. A. Ayon, R. Braff, C. C. Lin, H. H. Sawin, and M. A. Schmidt, "Characterization of a Time Multiplexed Inductively Coupled Plasma Etcher", J.Electrochem. Soc., 146, 339 (1999).

21. M. W. Losey, M. A. Schmidt, and K. F. Jensen. "A Micro Packed-Bed Reactor for Chemical Synthesis", in 3rd International Conference on Microreaction Technology. 1999. Frankfurt, Springer, Berlin (1999).

22. M. W. Losey, S. Isogai, M. A. Schmidt, and K. F. Jensen. "Microfabricated Devices for Multiphase Catalytic Processes", in 4 International Conference on Microreaction Technology. 2000. Atlanta, GA, AIChE, New York (2000), 416-422.

23. L. A. Christel, K. Petersen, W. McMillan, and M. A. Nortrup. "Nucleic Acid Concentration and PCR for Diagnostic Applications", in MlAS '98 Workshop. 1998. Banff, Canada, Kluwer Academic Publishers, Dordrecht (1998), 277-280.

24. T. Floyd, M. A. Schmidt, and K. F. Jensen. "Towards Integration of Chemical Detection for Liquid Phase Microchannel Reactors", in 4th International Conference on Microreaction Technology. 2000. Atlanta, GA, AIChE, New York (2000), 416-422.

25. S. L. Firebaugh, K. F. Jensen, and M. A. Schmidt. "Miniaturization And Integration of Photoacoustic Detection With a Microfabricated Chemical Reactor System", in $\mu T A S$ 2000 Workshop. 2000. Twente, The Netherlands, Kluwer Academic Publishers, Dordrecht, The Netherlands (2000).

26. J. J. Jackmann, T. Floyd, M. A. Schmidt, and K. F. Jensen. "Development of Methods for On-Line Chemical Detection With Liquid-Phase Microchemical Reactors Using Conventional and Unconventional Techniques" in $\mu T A S 2000$ Workshop. 2000. Twente, The Netherlands, Kluwer Academic Publishers, Dordrecht, The Netherlands (2000).

27. M. O. Heuschkel, L. Guerin, B. Buisson, D. Bertrand, and P. Renaud, "Buried Microchannels In Photopolymer For Delivering of Solutions To Neurons In a Network," Sensors and Actuators B-Chemical, 48, 356 (1998).

28. MEMCAD, Microcosm Technologies, Inc., 5511 Capital Center Dr., Suite 104, Raleigh, NC 27606.

29. $C F D-A C E$, CFD Research Corporation, 215 Wynn Dr., Huntsville, AL 35805.

30. I.-M. Hsing, R. Srinivasan, M. P. Harold, K. F. Jensen, and M. A. Schmidt, "Simulation of Micromachined Chemical Reactors for Heterogeneous Partial Oxidation Reactions," Chem. Eng. Sci., 55, 1 (2000).

31. D. J. Quiram, I.-M. Hsing, A. J. Franz, R. Srinivasan, K. F. Jensen, and M. A. Schmidt. "Characterization of Microchemical Systems Using Simulations", in 2 nd International Conference on Microreaction Technology. New Orleans, LA, AIChE, New York (1998) 205-10.

32. D. J. Quiram, J. F. R. Patrick L. Mills, M. D. Wetzel, J. M. Ashmead, T. M. Delaney, D. J. Kraus, J. S. McCracken, K. F. Jensen, and M. A. Schmidt. "Package Level Integration of Silicon Microfabricated Reactors to Form a Miniature Reactor Test System", in Technical Digest of the 2000 SolidState Sensor and Actuator Workshop. 2000. Hilton Head Isl., $\mathrm{SC}$, Transducer Research Foundation (2000). 